\title{
Spatio-spectral encoding of fringes in optical long-baseline interferometry
}

\section{Example of the 3T and 4T recombining mode of VEGA/CHARA}

\author{
D. Mourard ${ }^{1}$, Ph. Bério ${ }^{1}$, K. Perraut ${ }^{2}$, R. Ligi ${ }^{1}$, A. Blazit ${ }^{1}$, J. M. Clausse ${ }^{1}$, N. Nardetto ${ }^{1}$, A. Spang ${ }^{1}$, I. Tallon-Bosc ${ }^{3}$, \\ D. Bonneau ${ }^{1}$, O. Chesneau ${ }^{1}$, O. Delaa ${ }^{1}$, F. Millour ${ }^{1}$, P. Stee ${ }^{1}$, J. B. Le Bouquin ${ }^{2}$, T. ten Brummelaar ${ }^{4}$, C. Farrington ${ }^{4}$, \\ P. J. Goldfinger ${ }^{4}$, and J. D. Monnier ${ }^{5}$ \\ 1 Laboratoire Fizeau, OCA/UNS/CNRS UMR6525, Parc Valrose, 06108 Nice Cedex 2, France \\ e-mail: denis.mourard@oca.eu \\ 2 UJF-Grenoble 1/CNRS-INSU, Institut de Planétologie et d'Astrophysique de Grenoble (IPAG) UMR 5274, 38041 Grenoble, France \\ 3 UCBL/CNRS CRAL, 9 avenue Charles André, 69561 Saint Genis Laval Cedex, France \\ ${ }^{4}$ CHARA Array, Mount Wilson Observatory, 91023 Mt Wilson CA, USA \\ 5 University of Michigan, Ann Arbor, Michigan 48109-1090 MI, USA
}

Received 29 March 2011 / Accepted 9 May 2011

\section{ABSTRACT}

\begin{abstract}
Context. One of the main challenges of optical stellar interferometers is to increase the number of telescopes in the recombining unit to provide a larger number of measurements and an improved imaging capability. At the same time there is a need to preserve the spectroscopic capabilities, which leads to complex recombining schemes that may inhibit development.

Aims. We describe the possibilities of combining the spatial and spectral encoding of fringes for the design of more compact beam combiners and for minimizing the number of pixels that must be read.

Methods. We establish the formalism of the spatio-spectral fringe encoding, discuss general applications, and describe an implementation in the 3T/4T observing mode of the VEGA (Visible spEctroGraph and polArimeter) instrument installed at the coherent focus of the CHARA Array located on Mt Wilson in California. We finally present the science cases made possible by this instrumental implementation in the case of VEGA/CHARA.

Results. We demonstrate the interest in implementing an optimized spatio-spectral encoding of fringes in a multi-telescope beam combiner. On-sky results, obtained with the 3T mode of the VEGA combiner are presented. At visible wavelengths and with the hectometric baselines of CHARA, sub-mas stellar diameters could be determined with a precision of a few percent with a spectral resolution of 5000. Our first estimates of closure phase show that accuracies better than 1 degree can be achieved.

Conclusions. The first on-sky results obtained with the 3T-4T VEGA instrument using spatio-spectral fringe encoding show the validity of using this principle in the design of future complex beam combiners.
\end{abstract}

Key words. instrumentation: interferometers - instrumentation: spectrographs - methods: data analysis - stars: fundamental parameters

\section{Introduction}

Since the study of Labeyrie (1975), optical interferometry has been developed with spectroscopic capabilities to both relax the coherencing limits of white-light observations and increase the accessible scientific information. Combining spectral capabilities with interferometric measurements has led to important applications in stellar physics, such as the kinematical analysis of circumstellar environments or the chromatic study of stellar surfaces (Chiavassa et al. 2010). The need to combine more than two telescopes is of the utmost importance to achieve a higher spatial resolution analysis and permit accurate image reconstruction. A challenge is therefore to be able to combine three, four, or more telescopes and preserve the spectroscopic capacities as much as possible so as to achieve polychromatic imaging of stellar surfaces and/or environments. Interferometric beam-combination can follow co-axial or multi-axial optical approaches. In the latter case and for more than two telescopes, it is mandatory to optimize the detector pixel number while respecting both spectral and spatial sampling of fringe patterns.
One way of satisfying this condition is to combine spatio-OPD (optical path difference) coding of spectrally dispersed fringes as originally suggested for a space interferometer (Vakili \& Koechlin 1989). These authors demonstrated by means of a numerical simulation the possibility of retrieving the different high frequency information even in the case of a linear redundant configuration. In 2006, Le Bouquin \& Tatulli (2006) studied the possibility and efficiency of reducing the number of pixels in the case of multi-axial single mode interferometry through a partially redundant output pupil configuration.

The Center for High Angular Resolution Astronomy (CHARA) of the Georgia State University operates an optical interferometric array located at the Mount Wilson Observatory that consists of six one meter telescopes placed in pairs along the arms of a Y-shaped array. It yields 15 baselines ranging from 34 to $331 \mathrm{~m}$. Operating in the near-infrared with CLASSIC (ten Brummelaar et al. 2005), CLIMB (Sturmann et al. 2010), FLUOR (Coude du Foresto et al. 2003), and MIRC (Monnier et al. 2008), and in the visible with PAVO (Ireland et al. 2008) 
and VEGA (Mourard et al. 2009), the CHARA array allows a maximum angular resolution of 1.3 and $0.3 \mathrm{~ms}$ of arc in the $K$ and $V$ band, respectively. The VEGA spectrograph is designed to sample the visible band from 0.45 to $0.85 \mu \mathrm{m}$ with spectral resolutions of 1700,5000 , and up to 30000 . It is equipped with two photon counting detectors that simultaneously study two different spectral bands at high and medium spectral resolution. The principle and performance of VEGA, in its two telescope mode, has been presented in Paper I (Mourard et al. 2009). Fringes with three and four telescopes were obtained in 2010.

This paper aims to present a way of combining more than two telescopes in a dispersed fringe mode and discuss initial on-sky results in 3T and 4T mode of VEGA as an example. Section 2 explains the principle of the spatio-spectral encoding of fringes. Section 3 is devoted to the application of this principle to VEGA 3T/4T mode and to possible future instruments. We also present the first measurements obtained with VEGA in 3T mode and show the way in which we compute closure phase estimators in this multi-mode/multi-telescope/multi-lambda beam combiner. Finally in Sect. 4 we present some of the new science cases made possible by the use of this new observing mode.

\section{Principle of optimized spatio-spectral encoding of fringes}

In the case of interferometers based on dispersed fringe mode, the pupils are linearly recombined in a direction perpendicular to the spectral dispersion. Non-redundant linear reconfigurations are usually chosen (AMBER, Petrov et al. 2007; or MIRC Monnier et al. 2008) to easily separate the fringes coming from different pairs of telescopes. In this section, we develop a general formalism describing dispersed fringe interferograms and we explain how redundant pupil reconfigurations could be used without losing any information.

\subsection{Formalism of the spatio-spectral encoding}

Assuming that the flux of each beam is identical, the intensity of the interferogram can be written as

$I(x, y)=n i(x, y)\left(1+\frac{2}{n} \sum_{i=1}^{n} \gamma_{i} \cos \left[2 \pi \sigma \chi_{i}+\phi_{i}\right]\right)$,

where $(x, y)$ represents the pixels in the image plane, $i(x, y)$ represents the image of an individual pupil, $n$ is the number of baselines, $\sigma$ is the wave number, $\chi_{i}$ is the optical path difference (hereafter OPD) of baseline $i$ and $\gamma_{i}$ and $\phi_{i}$ are, respectively, the modulus and phase of the complex fringe visibility for the baseline $i$, and $\chi_{i}$ is a function of $x$ and $y$ that will be quantified in Eq. (4). Figure 1 shows a simulated interferogram in the dispersed fringe mode.

The direction of dispersion is parallel to the $x$ axis such that we have

$\lambda=a x$,

where $a$ is proportional to the dispersion coefficient of the dispersive optics.

We assume that the spectral bandwidth (total width $\Delta \lambda$ ) is small with respect to the wavelength $\lambda$. Denoting $\lambda_{0}$ as the center of the spectral band (corresponding to the pixel $x_{0}$ ), one can write for any wavelength of the spectral band $\lambda=\lambda_{0}+a\left(x-x_{0}\right)$. Thus we could write

$\sigma=\frac{1}{\lambda} \approx \frac{1}{\lambda_{0}}\left(1-\frac{a(x-x 0)}{\lambda_{0}}\right) \approx 2 \sigma_{0}-\frac{a x}{\lambda_{0}^{2}}$,

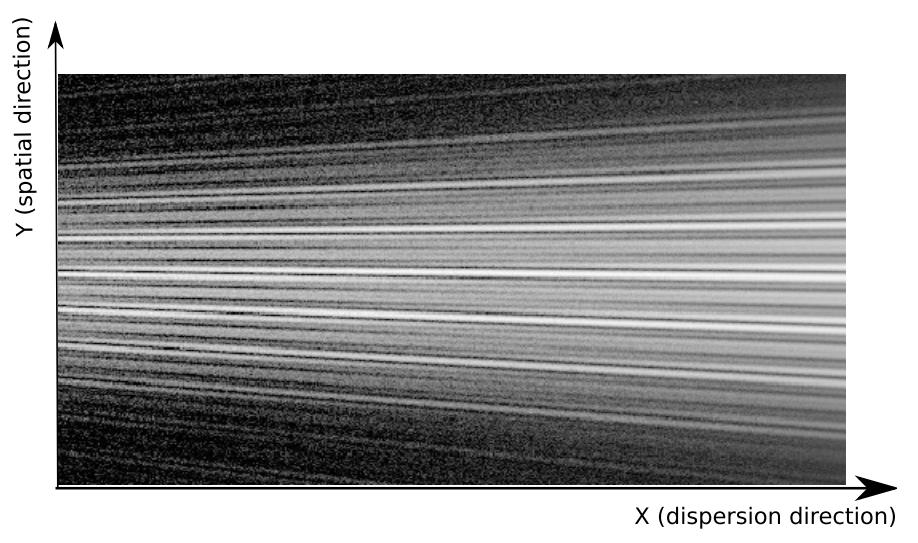

Fig. 1. Simulated interferogram in the dispersed fringe mode. Observations with four telescopes.

where $\lambda_{0}$ is the center of the spectral band.

Assuming a direction of dispersion perpendicular to the remapped pupil separation, the optical path difference $\chi_{i}$ could be written as a function of $y$ only. Therefore, we have

$\chi_{i}=\chi_{i}^{0}-b_{i} y$,

where $\chi_{i}^{0}$ is the OPD at the center of the image and $b_{i}$ depends on the pupil separation for baseline $i$ and the distance to the image plane. Substituting Eqs. (2)-(4) into Eq. (1) gives

$I(x, y) \approx n i(x, y)$

$$
\times\left(1+\frac{2}{n} \sum_{i=1}^{n} \gamma_{i} \cos \left[2 \pi\left(\chi_{i}^{0}-b_{i} y\right)\left(2 \sigma_{0}-\frac{a x}{\lambda_{0}^{2}}\right)+\phi_{i}\right]\right),
$$

which could also be written, using Eq. (3) for the term in $y$, as

$$
\begin{aligned}
I(x, y) \approx & n i(x, y) \\
& \times\left(1+\frac{2}{n} \sum_{i=1}^{n} \gamma_{i} \cos \left(4 \pi \chi_{i}^{0} \sigma_{0}+\phi_{i}-2 \pi \frac{a \chi_{i}^{0}}{\lambda_{0}^{2}} x-2 \pi b_{i} \sigma y\right)\right) .
\end{aligned}
$$

The Fourier transform $F T(I)(u, v)$ of such a fringe pattern shows peaks at high frequencies. As shown in Mourard et al. (2009), we estimate the energy of these high frequency peaks in order to estimate the squared visibility. $F T(I)(u, v)$ can be written as

$F T(I)(u, v)=n F T(i)(u, v) \otimes P(u, v)$

where

$$
\begin{aligned}
P(u, v) \approx 1 & +\frac{1}{n} \sum_{i=1}^{n} \gamma_{i} \mathrm{e}^{\mathrm{i}\left[\phi_{i}+4 \pi \chi_{i}^{0} \sigma_{0}\right]} \delta\left(u-a \sigma_{0}^{2} \chi_{i}^{0}\right) \delta\left(v+b_{i} \sigma\right) \\
& +\frac{1}{n} \sum_{i=1}^{n} \gamma_{i} \mathrm{e}^{-\mathrm{i}\left[\phi_{i}+4 \pi \chi_{i}^{0} \sigma_{0}\right]} \delta\left(u+a \sigma_{0}^{2} \chi_{i}^{0}\right) \delta\left(v-b_{i} \sigma\right) .
\end{aligned}
$$

If we take into account the sampling of the image, the coordinates of the high frequency peaks in pixels are

$\left(U_{p, i}, V_{p, i}\right) \approx\left(a \sigma_{0}^{2} \chi_{i}^{0} \Delta x,-b_{i} \sigma \Delta y\right)$,

where $(\Delta x, \Delta y)$ represents the total field in the source image. The final expression of $\left(U_{p i}, V_{p i}\right)$ is

$\left(U_{p i}, V_{p i}\right) \approx\left(\frac{\chi_{i}^{0} \Delta \lambda}{\lambda_{0}^{2}},-\frac{b_{i} \Delta y}{\lambda}\right)$.

This expression is identical to the one given by Koechlin et al. (1996) for the case of two telescopes. 


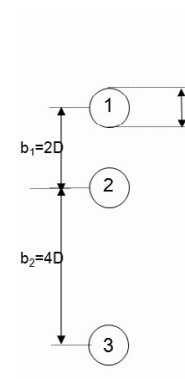

Pupil plane
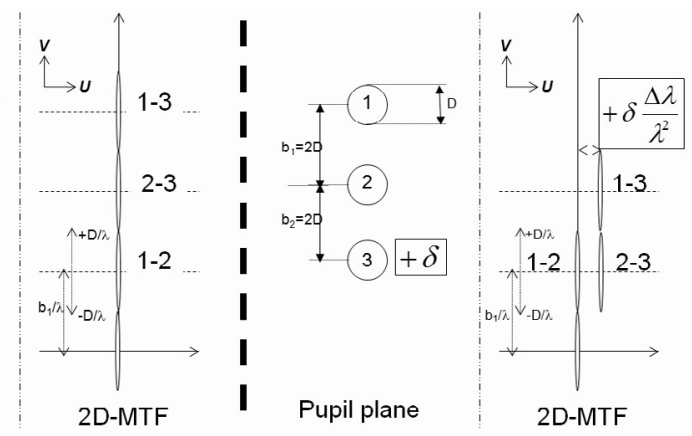

Fig. 2. Schematic representation of a simple 3T non-redundant (left) and redundant (right) linear configuration for a dispersed fringe instrument. For each case, we show the pupil plane at the entrance of the spectrograph and the 2D Modulation Transfer Function (MTF). By adding an optical path difference $(\delta)$ on one of the pupil in the redundant case, one can separate the fringe signals in the MTF plane despite the redundancy.

\subsection{Constraints for $V^{2}$ measurements}

In the case of non-redundant reconfigurations, the separation between two pupils is unique regardless of the number of telescopes (see Fig. 2)

$b_{i} \neq b_{j} \quad \forall i \neq j$.

The high frequency peaks of $F T(I)(u, v)$ are formed at different spatial frequencies in the $v$ direction so that the fringes could be locked onto the zero OPD for each baseline $\left(U_{p i}=0\right)$. In the case of redundant pupil reconfigurations, the pupil separation between two pupils can be identical for several baselines (see Fig. 2). Thus high frequency peaks of $\tilde{I}(u, v)$ are formed at the same spatial frequencies in the $v$ direction. However, it is possible to separate these peaks in the $u$ direction by placing the fringes at a different OPD for each baseline. Of course, these OPDs must be small with respect to the coherence length to avoid any loss of contrast. The idea is to reduce the overall size of the optics for $3 \mathrm{~T}$ operation from $7 * D$ (non redundant case) to $5 * D$ and the required number of pixels by the same amount. Figure 3 illustrates this redundant pupil reconfiguration principle by showing spectral densities of VEGA dispersed fringes in the case of observations with three and four telescopes. For example in the case of four telescopes, we can easily see a group of three high frequency peaks and a group of two high frequency peaks with the same coordinates in the vertical direction ( $v$ direction) but separated in the horizontal direction ( $u$ direction). The sixth high frequency peak, alone at its vertical coordinate, corresponds to the largest output baseline.

If we consider the quantity $U_{p}$ of Eq. (10), we can easily deduce a general rule for correctly separating the different fringe signals with the same $V_{p}$ spatial frequency. We define $\delta \lambda$ as the spectral width of an elementary spectral channel. Thus, $N_{\mathrm{ch}}=\frac{\Delta \lambda}{\delta \lambda}$ is the number of spectral channels and $l_{\mathrm{c}}=\frac{\lambda^{2}}{\delta \lambda}$ is the coherence length of the dispersed fringes. We can then write

$\chi_{i}^{0}=\frac{U_{p i}}{N_{\mathrm{ch}}} * l_{\mathrm{c}}$.

For a linear redundant configuration of $n$ telescopes, the first $V_{p}$ frequency will be shared by $(n-1)$ baselines and separating them in the other direction $\left(U_{p}\right)$ is only possible if the number of spectral channels is larger than the number $N$ of telescopes or ideally two times the number of telescopes to avoid any overlapping between the different fringe signals. We can then write this important condition for a correct implementation of the spatiospectral encoding as:

$N_{\text {ch }} \geq \sim 2 * N$.

\subsection{Restoring the spectral resolution}

As a consequence of the previous formalism, one can see that computing the Modulation Transfer Function on a single spectral channel will mix all fringe signals with the same $V_{p}$ frequency. However, the spectral resolution could be restored thanks to the differential analysis of data. Computing the cross-spectrum between a small spectral channel and the whole spectral band allows one to extract the complex differential visibility as is done in the standard VEGA/CHARA data reduction pipeline (Mourard et al. 2009).

Referring to Eq. (7), we can write, using the gate function $\Pi$, the expression of the cross spectrum analysis between a large spectral channel $(\Delta x)$ and a narrow one $(\delta x)$

$F T\left(I_{\Delta x} \otimes I_{\delta x}\right)=F T\left(I_{\Delta x} \otimes\left(I_{\Delta x} \times \Pi_{\delta x}\right)\right)$,

where the symbol FT indicates the Fourier transformation. This expression can be written as

$F T\left(I_{\Delta x} \otimes I_{\delta x}\right)=F T\left(I_{\Delta x}\right) \times\left[F T\left(I_{\Delta x}\right) \otimes F T\left(\Pi_{\delta x}\right)\right]$.

It is clear that the convolution of $F T\left(I_{\Delta x}\right)$ by the term $F T\left(\Pi_{\delta x}\right)$ and then the filtering by the support of $F T\left(I_{\Delta x}\right)$ will mix the information coming from the different spatio-spectral frequencies $u$ of the same spatial frequency $v$. However, one can place constraints on the width of the small spectral band so that the effect of $F T\left(\Pi_{\delta x}\right)$ is negligible or it is possible to take this effect into account when defining the differential visibility estimators. We now consider the simple case of a three telescope linear redundant configuration as shown in Fig. 2. This configuration exhibits two spatial frequencies related to the baselines $b$ and $2 b$. If we consider the spatial frequency corresponding to the baseline $b$, we have two separate spatio-spectral frequencies corresponding to the delays $\chi_{1,2}^{0}$ set for the two redundant baselines. From Eq. (15), we can calculate the value $I_{1}$ and $I_{2}$ of the cross-spectrum at the frequencies $\left[\sigma_{0}^{2} \chi_{1}^{0} \Delta \lambda,-b \sigma_{0} \Delta y\right]$ and $\left[\sigma_{0}^{2} \chi_{2}^{0} \Delta \lambda,-b \sigma_{0} \Delta y\right]$ to be

$I_{1}=\gamma_{1, \Delta \lambda} \gamma_{1, \delta \lambda} \mathrm{e}^{\mathrm{i}\left[\phi_{1, \Delta \lambda}-\phi_{1, \delta \lambda}\right]}+\gamma_{1, \Delta \lambda} \gamma_{2, \delta \lambda} \mathrm{e}^{\mathrm{i}\left[\phi_{1, \Delta \lambda}-\phi_{2, \delta \lambda}\right]} \operatorname{sinc}(\alpha)$

$I_{2}=\gamma_{2, \Delta \lambda} \gamma_{2, \delta \lambda} \mathrm{e}^{\mathrm{i}\left[\phi_{2, \Delta \lambda}-\phi_{2, \delta \lambda}\right]}+\gamma_{2, \Delta \lambda} \gamma_{1, \delta \lambda} \mathrm{e}^{\mathrm{i}\left[\phi_{2, \Delta \lambda}-\phi_{1, \delta \lambda}\right]} \operatorname{sinc}(\alpha)$

where

$\alpha=\pi a \sigma_{0}^{2}\left(\chi_{1}^{0}-\chi_{2}^{0}\right) \Delta x \delta x=\frac{\pi\left(\chi_{1}^{0}-\chi_{2}^{0}\right) \Delta x}{\lambda_{0}^{2}} \delta \lambda$.

We note that $\Delta x$ represents the number of pixels in the spectral direction of the large spectral band $\Delta \lambda$. These equations show that at the frequency $\left[\sigma_{0}^{2} \chi_{1}^{0} \Delta \lambda,-b \sigma_{0} \Delta y\right]$, the differential measurement of baseline $1\left(\gamma_{1, \Delta \lambda} \gamma_{1, \delta \lambda} \mathrm{e}^{\mathrm{i}\left[\phi_{1, \Delta \lambda}-\phi_{1, \delta \lambda}\right]}\right)$ is corrupted by a term coming from baseline $2\left(\gamma_{1, \Delta \lambda} \gamma_{2, \delta \lambda} \mathrm{e}^{\mathrm{i}\left[\phi_{1, \Delta \lambda}-\phi_{2, \delta \lambda}\right]}\right)$ but attenuated by the sinc function. The symmetric situation occurs at the second frequency $\left[\sigma_{0}^{2} \chi_{2}^{0} \Delta \lambda,-b \sigma_{0} \Delta y\right]$. If we wish to limit the contamination between peaks at a level of $1 \%, \alpha$ should be greater than 100. Therefore, we can define a constraint on the narrow spectral bandwidth given by

$\delta \lambda \geq \delta \lambda_{\min }=\frac{100 \lambda_{0}^{2}}{\pi\left|\chi_{1}^{0}-\chi_{2}^{0}\right| \Delta x}$. 

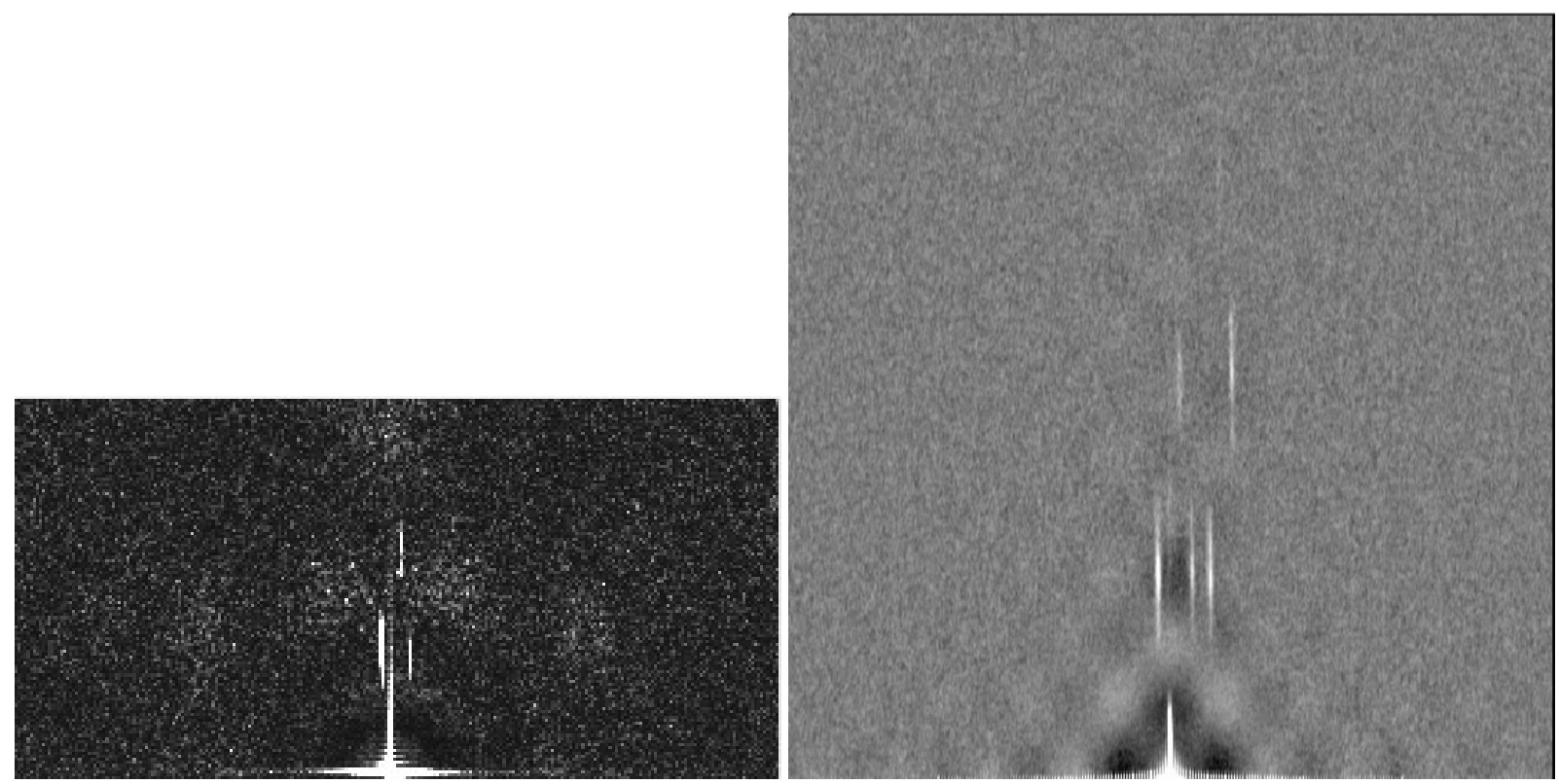

Fig. 3. Spatio-spectral densities of VEGA dispersed fringes in 3T mode (left) with CLIMB used as IR group delay tracking and in 4T mode (right) with MIRC used as IR group delay tracking. Axes correspond to the $(u, v)$ coordinates defined in Eq. (6): $u$ is the horizontal direction, $v$ the vertical one. The three (respectively sic) high-frequency fringe signals are clearly separated in this $2 \mathrm{D}$ spectral density for the $3 \mathrm{~T}$ (respectively 4T) mode.

This constraint can induce a degradation of the actual spectral resolution during the data processing but this disadvantage can also be removed by the complete solution of the system of equations described in Eq. (16). For example, if we compute $\delta \lambda_{\text {min }}$ in the case of the differential processing presented in Sect. 3.5 (where $\lambda_{0}=735 \mathrm{~nm},\left|\chi_{1}^{0}-\chi_{2}^{0}\right|=450 \mu \mathrm{m}$ and $\Delta x=1816 \mathrm{px}$ ), we obtain $\delta \lambda_{\min }=0.02 \mathrm{~nm}$. This value is well below the medium spectral resolution of VEGA.

\subsection{Interest for future spectro-interferometric instruments}

We now consider the case for a possible future dispersed fringe near-infrared instrument recombining $N_{\mathrm{t}}=6$ or 8 telescopes and we compare its performance with or without the use of the spatio-spectral encoding. The main issue is of course the smaller number of pixels that have to be read, which leads to a reduction in both the readout noise and the total overhead. If we consider the linear non-redundant configuration proposed in Le Bouquin \& Tatulli (2006) for 6 and 8 telescopes, we can calculate that the output pupil extends over 36 (respectively 76) times the individual diameter for 6 (respectively 8 ) telescopes. The spatiospectral encoding allows us to reduce these numbers to 12 (respectively 16) times the individual diameter. Thus, the gain in the number of pixels is 3 (respectively 4.75 ) in 6T (respectively $8 \mathrm{~T})$ mode.

It is clear that following recent progresses in developing very low noise infrared detectors, this gain will not be as high but one has to keep in mind that an accurate calculation should also take into account the observing efficiency that includes the percentage of time on sky versus that on reading the detector. Hence the implementation of this optimized encoding will certainly bring important improvements in terms of limiting magnitude of future multi-axial instruments or fringe tracker devices.
Table 1. Optimal characteristics of the spatio-spectral encoding of the VEGA-3T/4T modes in medium spectral resolution.

\begin{tabular}{lccl}
\hline \hline MODE & $N_{\mathrm{ch}}$ & $l_{\mathrm{c}}(\mathrm{mm})$ & OPD offsets $(\mu \mathrm{m})$ \\
\hline 3T & 200 & \pm 1.8 & $0-100+150$ \\
$4 \mathrm{~T}$ & 200 & \pm 1.8 & $0-100+150+250$ \\
\hline
\end{tabular}

Notes. The last column indicates the typical OPD offsets for pupils 1-23 or 1-2-3-4. The actual implementation could be different, thanks to the residual cophasing errors between VEGA and the infrared instruments.

\section{On sky validation: VEGA-3T first measurements and performance}

\subsection{Context of VEGA}

The VEGA spectrograph (Mourard et al. 2006, 2008) was originally developed, in terms of optics, to accept three beams linearly arranged in a non-redundant way, as presented in Fig. 2left. To preserve the correct sampling in the detector plane, we considered adding anamorphosis optics or changing the magnification, which caused a reduction in either the field of view or width of the spectral band. Thanks to improved algorithms developed for photon counting detectors (Blazit et al. 2008), we demonstrated the possibility of simply improving the spatial resolution in the image plane by a factor of four through sub-pixel localization of photo-events. By adopting the new principle of spatio-spectral encoding, it was then possible to simultaneously recombine not only three telescopes but even four in a high redundant mode. The interest lies in the huge improvement in the measurement's output with three squared visibilities and one closure phase in $3 \mathrm{~T}$ mode and six squared visibilities and three independent closure phases in 4T mode (see Fig. 3).

Table 1 presents the main characteristics of VEGA/CHARA 3T-4T modes used in the medium resolution mode $(R=6000)$. 
D. Mourard et al.: Spatio-spectral encoding of fringes in optical long-baseline interferometry

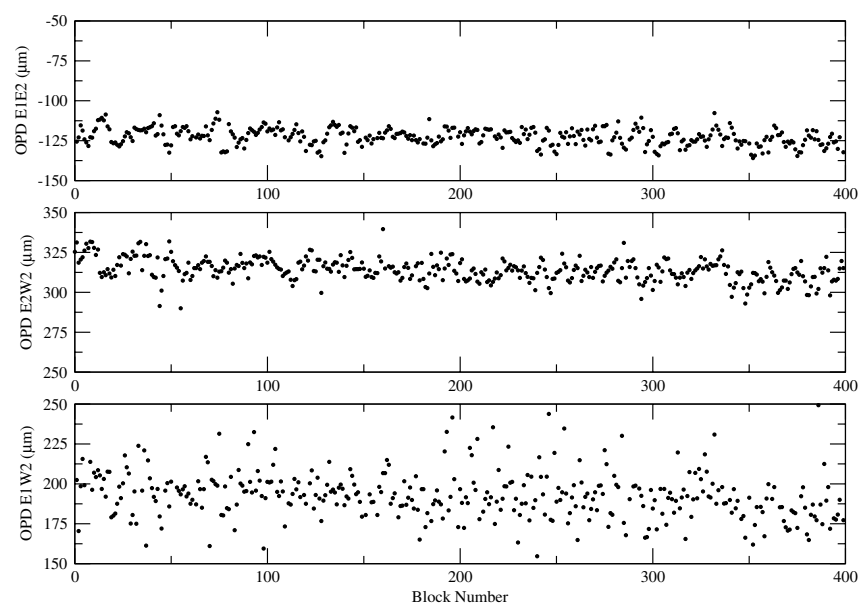

Fig. 4. Observation of the calibrator star HD 55185 with VEGA with three telescopes (E1E2W2) at medium spectral resolution $(\Delta \lambda=30 \mathrm{~nm}$ centered at $\lambda=735 \mathrm{~nm}$ ). The tracking of the fringes is done in the nearinfrared with the instrument CLIMB. Plots represent the OPD residuals measured on VEGA data for the three baselines. The OPD estimates are done each second (50 short exposures of $20 \mathrm{~ms}$ ). Note that, according to the principle described in Sect. 2, the fringes are not stabilized around null OPDs. For this specific experiment, we did not used the standard configuration as defined in Table 1.

\subsection{General remarks on the VEGA-3T and $-4 T$ observing modes}

$3 \mathrm{~T}$ and 4T operation, especially with CHARA, immediately leads to long baselines because of the telescope configuration. The more compact telescope triplet (S1S2W2) has already a baseline of $170 \mathrm{~m}$, which leads to low visibility measurements in the visible. The other triplets lead to very long baselines of up to $200 \mathrm{~m}$ and even $330 \mathrm{~m}$. Until mid-2010, we were only able to stabilize the VEGA coherence through a real-time processing of the science data allowing us to correct the offset of the delay lines. This mode prevents us, however, from an efficient groupdelay tracking at the needed rate of $0.1 \mathrm{~Hz}$ for a visibility below 0.3. In 2010, we succeeded in observing with VEGA, while the fringes were externally stabilized with the new CHARA infrared instrument called CLIMB (Sturmann et al. 2010) for 3T operations, and with MIRC (Monnier et al. 2008) for the first 4T VEGA light. With these external group delay trackers, we measured a typical residual jitter on the optical path difference of about 5 microns (see Fig. 4), which is well adapted to our high and medium spectral resolution modes. For this operation, we introduced differences in the internal offsets between VEGA and CLIMB or MIRC so that fringes are stabilized in the infrared around a null optical path difference and around non-null offsets in the visible to correctly apply the principle of spatio-spectral encoding as described in Sect. 2.

\subsection{Squared visibility estimation}

Mourard et al. (2009) described squared visibility estimations in the case of observations with two telescopes. In the case of three or four telescope observations, we used exactly the same estimator. hence, we measured the energy of each high frequency peak of the power spectrum calibrated by the energy of the low frequency peak. In the case of 3(4) telescopes, we estimated 3(6) squared visibilities.

Here, we illustrate the quality of observations for three telescopes and the data reduction by deriving the angular diameter

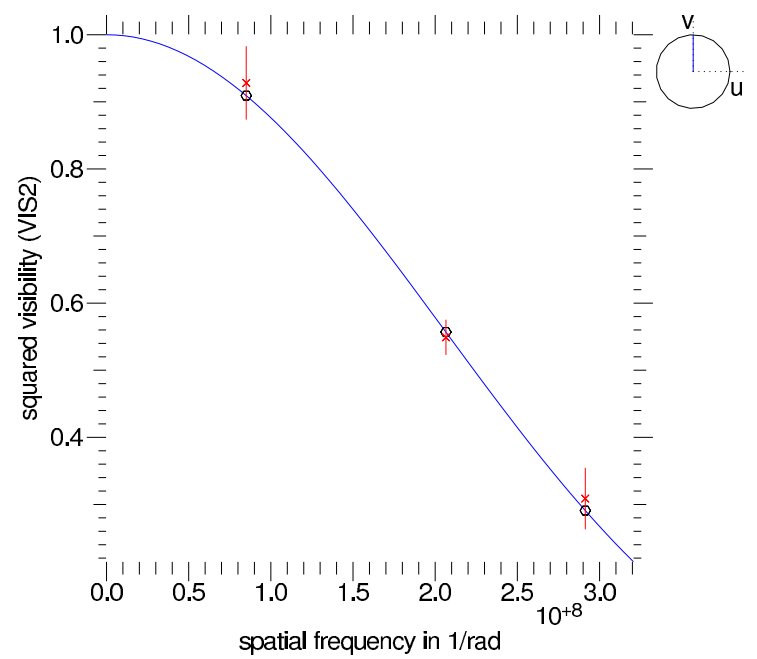

Fig. 5. Fit of a uniform disc model to the squared visibilities measured with VEGA on HD 55185. VEGA measurements are plotted with crosses and error bars and the model is plotted with circle. The model corresponds to a uniform disk angular diameter of $\phi_{\mathrm{HD}} 55185=$ $0.474 \pm 0.014$ mas.

of HD 55185. We used HD 46487 as a calibrator. The observations in 2010 October 16 were performed using the telescopes E1E2W2 and the group-delay sensor CLIMB, and were of medium spectral resolution around $735 \mathrm{~nm}$, and consisted of $400 \mathrm{~s}$ of total observation time corresponding to 20000 short exposures of $20 \mathrm{~ms}$. In the data reduction, we selected a spectral band of $\Delta \lambda=30 \mathrm{~nm}$ centered on $\lambda=735 \mathrm{~nm}$. We assumed that the uniform disk (UD) angular diameter of the calibrator is $\phi_{\mathrm{HD} 46487}=0.18 \pm 0.01$ mas (estimated value given by SearchCal tool $^{1}$ of the JMMC, Bonneau et al. 2006a). We used the LitPro ${ }^{2}$ software (Tallon-Bosc et al. 2008) to fit a uniform disc model to the squared visibilities measured on the three baselines and estimated and angular diameter of HD 55185 (see Fig. 5). We found $\phi_{\mathrm{HD}} 55185=0.474 \pm 0.014$ mas.

We used surface brightness relations to obtain an independent estimate of the angular diameter (Kervella et al. 2004). We found 0.478 mas and 0.476 mas from $(V-R)$ and $(B-V)$, respectively. Our result is therefore in very good agreement with these photometric estimations.

Our determination, after conversion into a limb-darkened diameter, is combined with the recent determination of the paral$\operatorname{lax}(8.49 \pm 0.17$ mas, van Leeuwen 2007) to obtain an estimation of the effective temperature of HD 55185. This leads to $9488 \pm 163 \mathrm{~K}$, which is coherent for a A2V star. Our result illustrates the capability of VEGA for measuring sub-mas angular diameters of star with a precision of a few percent $(3 \%$ in the case of HD 55185) and obtain a very reliable estimate of effective temperature.

\subsection{Closure phase estimation}

The problem of obtaining the most reliable estimate of the closure phase has been examined by a number of authors. Cornwell (1987) showed that the closure phase is equal to the phase of the bispectrum (or triple product) and Woan \& Duffett-Smith (1988) showed that averaging the bispectrum is the best way to estimate the closure phase if the visibility phases cannot be recovered.

\footnotetext{
1 Available at http://www . jmmc . fr/searchcal

2 Available at http://WwW. jmmc.fr/litpro
} 

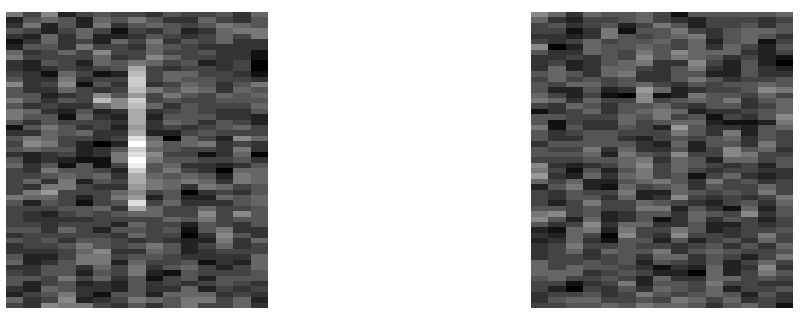

Fig. 6. Bispectrum averaged over 250 short exposures of $20 \mathrm{~ms}$ (observation of the calibrator star HD 55185). Left: real part of the bispectrum, right: imaginary part of the bispectrum.

The bispectrum is a complex valued function defined as:

$D^{(3)}\left(u_{1}, u_{2}, v_{1}, v_{2}\right)=\tilde{I}\left(u_{1}, v_{1}\right) \tilde{I}\left(u_{2}, v_{2}\right) \tilde{I}^{*}\left(u_{1}+u_{2}, v_{1}+v_{2}\right)$,

where $\tilde{I}(u)$ is the Fourier transform of the fringe pattern and $\tilde{I}^{*}$ denotes the complex conjugate of $\tilde{I}$. The argument of the bispectrum is the closure phase $\Delta \phi$

$\arg \left[D^{(3)}\left(u_{1}, u_{2}, v_{1}, v_{2}\right)\right]=\Delta \phi=\theta_{12}+\theta_{23}-\theta_{13}$,

where $\theta_{i j}$ is visibility phase of baseline $i j$.

The dimension of the bispectrum is always twice that of the power spectrum. In the case of VEGA, the bispectrum will then be four dimensional as presented in Eq. (19). Because the small number of telescopes (three or four) provides only a small number of closure triangles, it is only necessary to calculate the bispectrum on a small number of points. In the case of three telescopes, the bispectrum is calculated around $\left(U_{p 1}, U_{p 2}, V_{p 1}, V_{p 2}\right)$ as defined in Eq. (10).

Figure 6 shows the real and imaginary parts of the bispectrum averaged over 250 short exposures of $20 \mathrm{~ms}$ around the frequencies $\left(U_{p 1}, U_{p 2}, V_{p 1}, V_{p 2}\right)$. The averaged bispectrum has been projected on the plane defined by the axis $\left(u_{1}, v_{1}\right)$ in order to ease the visualization of this four-dimensional function. We show a signal in the real part of the averaged bispectrum whereas no signal is present in the imaginary part. This is of course expected since the star is unresolved and therefore the closure phase is zero.

Figure 7 presents closure phase measurements for two calibrator stars (HD 46487 and HD 55185). The observations were performed using three telescopes (E1E2W2) at medium spectral resolution. Each estimate corresponds to $5 \mathrm{~s}$ of observation and to a spectral band of $\Delta \lambda=30 \mathrm{~nm}$ centered at $\lambda=735 \mathrm{~nm}$. The mean and standard deviation of both series are

$\overline{\Delta \phi}_{\mathrm{HD} \mathrm{46487}}=-0.091 \pm 8.499^{\circ}$,
$\overline{\Delta \phi}_{\mathrm{HD} \mathrm{55185}}=0.776 \pm 5.625^{\circ}$.

No significant bias in the closure phase is detected from these estimates. The difference in the precision of the two sets is related to the photon noise, HD 55185 having a magnitude $m_{V}=4.14$ compared to $m_{V}=5.08$ for HD 46487. These first estimates of closure phase also show that precision better than $1^{\circ} \approx 20 \mathrm{mrad}$ can be achieved with VEGA by averaging phase closure estimates over several minutes (10 mn for HD 46487 for example).

\subsection{Differential measurements}

Spectro-interferometers such as VEGA permit us to determine the differential visibility and phase. These instruments have been
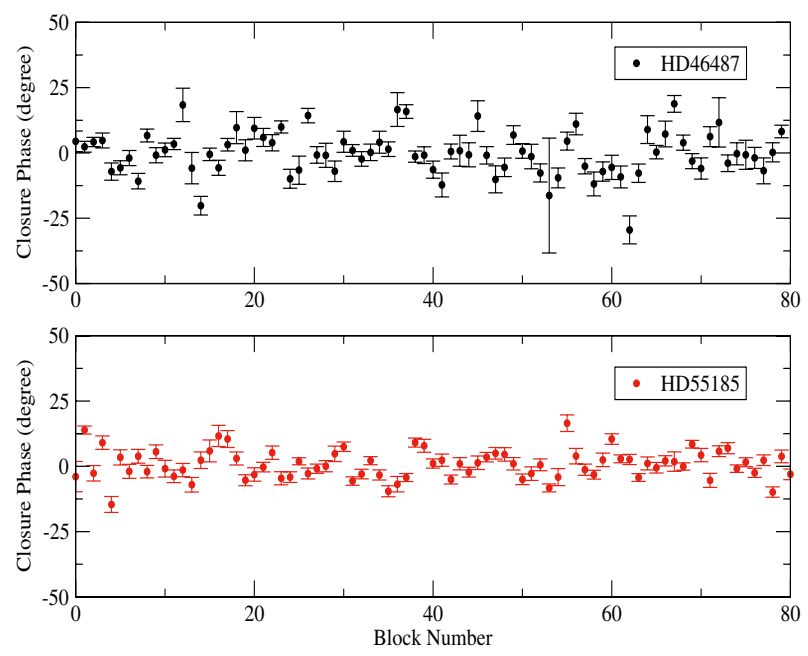

Fig. 7. Estimate of the closure phase of calibrator stars HD 46487 and HD 55185 with VEGA with three telescopes (E1E2W2) at medium spectral resolution $(\Delta \lambda=30 \mathrm{~nm}$ centered at $\lambda=735 \mathrm{~nm})$ and fringe tracking done with CLIMB. Each estimate corresponds to $5 \mathrm{~s}$ of observation (250 short exposures of $20 \mathrm{~ms}$ ).

intensively used, for example, to study the morphology and kinematic of circumstellar environments (Mourard et al. 1989; Berio et al. 1999; Meilland et al. 2007). A data reduction method was developed to derive these differential measurements for VEGA, which is described in detail in Mourard et al. (2009) for two telescope observations. In the case of three or four telescope observations, we use exactly the same method but for each high frequency peak of the cross-spectrum.

Here, we address the problem of the differential phases in the case of three telescope observations. In Fig. 8, we present measurements of differential phase for the observations of HD 55185 (see the description of observation in Sect. 3.3). We used a reference channel of $\Delta \lambda_{1}=30 \mathrm{~nm}$ centered at $\lambda_{1}=735 \mathrm{~nm}$ and a sliding narrow channel of $\Delta \lambda_{2}=0.6 \mathrm{~nm}$. We averaged the crossspectrum of 20000 short exposures of $20 \mathrm{~ms}$ (corresponding to $400 \mathrm{~s}$ of observation) in order to estimate the differential phase with a good signal to noise ratio (SNR). In theory, the differential phase curves should be constant and equal to zero regardless of the baseline (E1E2, E1W2, or E2W2), since the star is very partially resolved. However, we detect linear and/or quadratic trends for each baseline. The differential phase is corrupted by the OPD

$\Delta \phi_{12, i}=\theta_{\lambda_{1}, i}-\theta_{\lambda_{2}, i}+2 \pi\left(\frac{\chi_{i}\left(\lambda_{1}\right)}{\lambda_{1}}-\frac{\chi_{i}\left(\lambda_{2}\right)}{\lambda_{2}}\right)$,

where $\Delta \phi_{12, i}$ is the differential phase for the baseline $i, \theta_{\lambda_{j}, i}$ is the phase of the object at the wavelength $\lambda_{j}$ for the baseline $i$, and $\chi_{i}\left(\lambda_{j}\right)$ is the OPD of the baseline $i$. This OPD depends on the wavelength through the chromatism of the refractive index of air (Colavita et al. 2004; and Ciddor 1996)

$\chi_{i}(\lambda)=n(\lambda) L_{i}=(1+N(\lambda)) L_{i}=\chi_{i}+\delta \chi_{i}(\lambda)$

where $n(\lambda)$ is the refractive index of air, $N(\lambda)$ represents the chromatic part of $n(\lambda)$, and $L_{i}$ is the path difference in air for the baseline $i$. Although CHARA uses vacuum pipes for the beam transportation, the delay lines are in open air, thus the difference in the positions of delay lines introduced a difference in the path in air. In Eq. (23), $\chi_{i}$ is the static part of the OPD as described in Sect. 2 and $\delta \chi_{i}(\lambda)$ is the chromatic OPD. Hence, inserting 

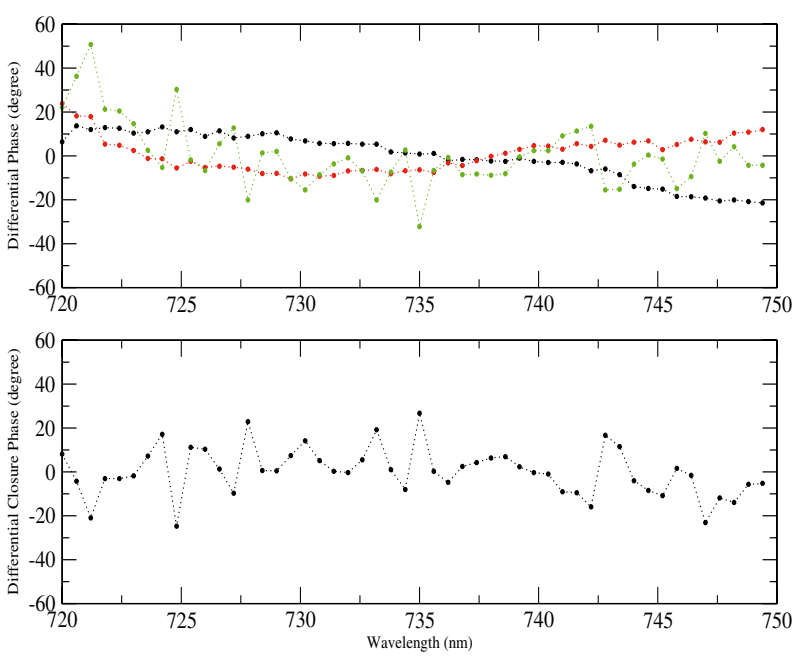

Fig. 8. Top: plots represent the differential phases in a narrow spectral band of $0.6 \mathrm{~nm}$ with respect to the wavelength (baseline E1E2 in black, E2W2 in red, E1W2 in green). Bottom: plot represents the differential closure phase deduced from the three individual differential phases. Each estimate corresponds to $400 \mathrm{~s}$ of observation of calibrator star HD 55185 using VEGA with three telescopes (E1E2W2) at medium spectral resolution and fringe tracking done with CLIMB. Individual error bars are not plotted for clarity. On baselines E1E2 and E2W2, the errors are at the level of $0.5^{\circ}$, whereas on the baseline E1W2 they are closer to $5^{\circ}$.

Eqs. (23) into (22) gives

$\Delta \phi_{12, i}=\theta_{\lambda_{1}, i}-\theta_{\lambda_{2}, i}+2 \pi \chi_{i}\left(\frac{1}{\lambda_{1}}-\frac{1}{\lambda_{2}}\right)+\phi_{\text {disp }, 12}$,

where the dispersive differential phase is

$\phi_{\text {disp }, 12}=2 \pi\left(\frac{\delta \chi_{i}\left(\lambda_{1}\right)}{\lambda_{1}}-\frac{\delta \chi_{i}\left(\lambda_{2}\right)}{\lambda_{2}}\right)$.

Hence, if the interferometer operates in vacuum and if the OPD is servoing to zero, the third and fourth terms in Eq. (24) disappear and the differential phase depends only on the phase of the object. In the case of VEGA, the OPDs of each baseline are servoing to a non-zero value and the delay lines operate in air. Therefore, the differential phases are corrupted by both the static OPD and the dispersive differential phase $\phi_{\text {disp, } 12}$, which explains the linear and/or quadratic trends of the curves presented in Fig. 8.

Extraction of the astrophysical signal requires the removal of these linear and/or quadratic trends. This is usually done by fitting and subtracting a low-order polynomial function or a model of the dispersive differential phase to the differential phase (Matter et al. 2010). The main drawback of this method is the loss of both the linear and/or quadratic terms in the astrophysical signal. For studies of circumstellar discs for example, it is not a major problem because the astrophysical signal is more complex and confined only to spectral lines. So the neighboring continuum could be used to remove the linear and/or quadratic trends. However, other astrophysical studies, such as binary stars, require us to measure signatures in the differential phase that are very close to linear or quadratic trends. In that case, the differential phase could not be used directly. We defined a new interferometric observable, which is the differential closure phase

$\Delta \phi_{12}^{(3)}=\Delta \phi_{12,1}+\Delta \phi_{12,2}-\Delta \phi_{12,3}$.

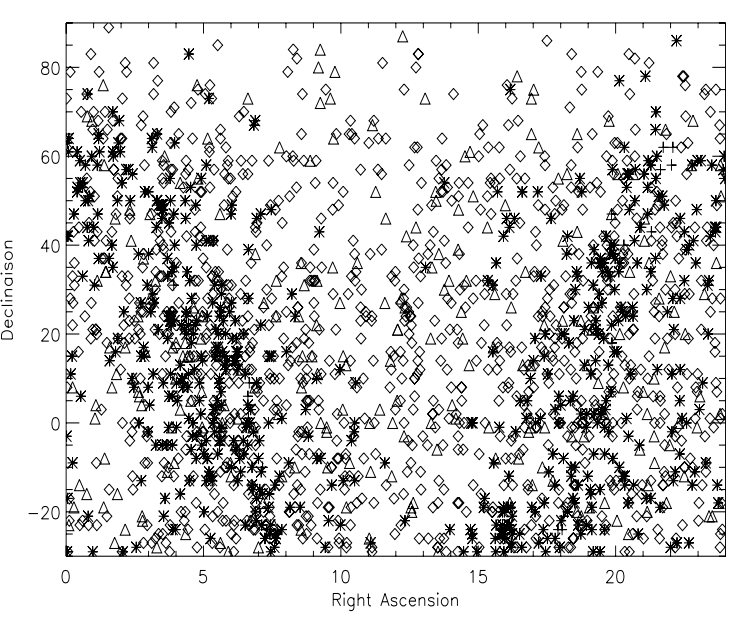

Fig. 9. Sky coverage of potential calibrator stars well adapted to VEGA/CHARA in 3T/4T mode with CLIMB as external IR group delay tracking. All these stars have a spectral type ranging from $\mathrm{O}$ (cross), B (star), A (diamond), to F (triangle).

It can easily be shown that this quantity does not depend on the OPD but is the difference in the classical closure phase of each spectral band

$\Delta \phi_{12}^{(3)}=\Delta \phi_{1}-\Delta \phi_{2}$,

where $\Delta \phi_{1}$ and $\Delta \phi_{2}$ are respectively the classical closure phase of the reference channel and of the narrow channel. Figure 8 (bottom plot) illustrates that the linear and/or quadratic trends due to the OPD have disappeared in the differential closure phase. The mean of the differential closure phase in one narrow channel is $\overline{\Delta \phi}_{12}^{(3)}=0.2 \pm 11^{\circ}$ all over the spectral band of $30 \mathrm{~nm}$.

\section{Discussion of possible VEGA/3T-4T science cases}

\subsection{T-4T operation issues}

Even if progress in fringe tracking allows fainter objects to be observed and/or lower visibilities to be recorded, this requires finding calibrators that are bright enough for both the scientific instrument and the fringe tracker. Moreover, using very long baselines makes the choice of calibrators much more difficult since lots of them are partially resolved. The calibrators must also be close to the scientific targets in terms of both distance and spectral type to avoid any bias in the calibrated interferometric observables. As an example, we selected calibrators among the 9500 stars (declination larger than $-30^{\circ}$ and magnitude $V$ brighter than 7.0) in the VEGA internal catalog by applying filters to parameters such as magnitude and diameter so as to fit the VEGA capabilities. Angular diameters were calculated using a spectrophotometric polynomial law based on the $V$ magnitude and the $(V-K)$ color index (Bonneau et al. 2006b). According to the current limiting magnitude, we considered only the stars brighter than 6.5 in the $V$ band and brighter than 7 in the $K$ band (for CLIMB that performs the group delay tracking). We identified 1866 potential calibrators in all parts of the sky with an angular diameter smaller than 0.4 mas providing a high visibility measurement even on the largest baselines. All of them are OBAF stars (Fig. 9). Figure 10 shows the histogram of the number of calibrators found per regions of sky of $1 \mathrm{~h}$ in right ascension and $5^{\circ}$ in declination. 


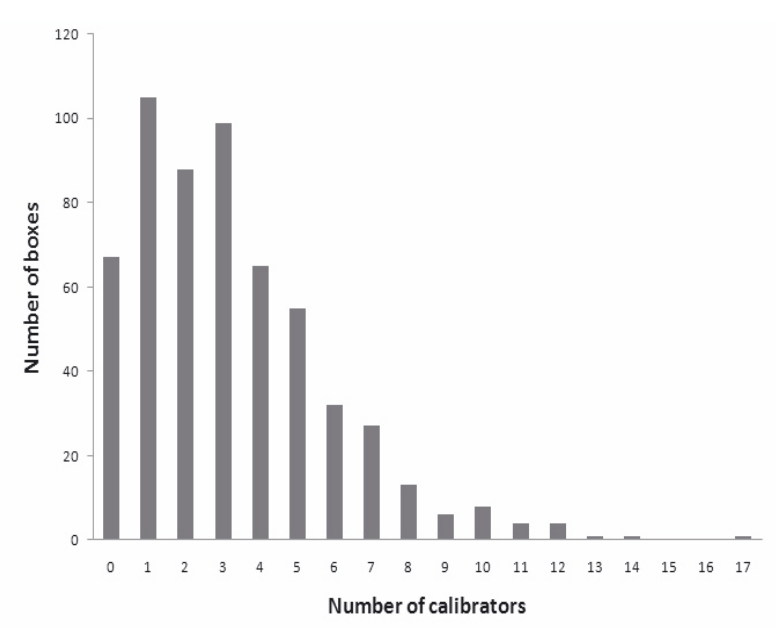

Fig. 10. Number of sky regions of $1 h$ in right ascension and $5^{\circ}$ in declination as a function of existing calibrators.

\subsection{Fundamental parameters}

Thanks to direct angular diameter measurements by optical interferometry combined with the bolometric flux, it is possible to derive the fundamental stellar parameters, such as the effective temperature, the luminosity, and the stellar radius. The determination of the angular diameter is based on visibility measurements that are directly linked to the Fourier transform of the object intensity distribution. For a single circular star, the visibility curve as a function of spatial frequency $B / \lambda$ (where $B$ is the interferometric baseline and $\lambda$ is the operating wavelength) is related to the first Bessel function, and contains a central lobe, that is almost only sensitive to the size of the object, and an ever decreasing series of side lobes, separated by nulls, which one observes with an increasing angular resolution. As a rule of thumb, the side lobes are sensitive to limb darkening and atmospheric structure but consist of very low visibilities. With VEGA, being able to measure very low visibilities will allow us to determine the angular diameters of stars of sub-mas sizes to an accuracy better than 2\% (Mourard et al. 2010). We limit ourselves to stars presenting an angular diameter smaller than 3.0 mas in order to be able to cover both the central lobe and the side lobes of the visibility function.

Moreover, using triplets of the longest baselines in the visible is a powerful means of determining accurate limb darkening laws throughout the HR diagram. Limb darkening is a function of the wavelength, and in particular limb darkening in a given spectral line depends upon the physical location in the atmosphere of the line-forming region of the element. Thus, an accurate measurement of the limb-darkening within spectral lines will allow us to study the temperature and density structure of the atmosphere, hence the strong interest in using multi-telescope combiners operating with dispersed fringes.

For VEGA, by adopting the same approach used for searching to calibrators and considering the standard VEGA squared visibility measurement accuracy of $2 \%$ and the CHARA longest baseline $(330 \mathrm{~m})$, we have determined the minimum measurable diameter for a given accuracy. This corresponds to 0.16 mas (resp. $0.12,0.075$ ) for a $1 \%$ (resp. $2 \%, 5 \%$ ) accuracy in the angular diameter determination. We have then determined that such limb-darkening studies will be possible for stars spread over nearly the whole Hertzsprung-Russell diagram (excluding compact objects) as illustrated in Fig. 11, and will result in a very significant improvement in the stellar atmosphere models (1D and 3D) of all stars.

\subsection{Towards chromatic imaging of stellar surfaces and complex environments}

Visibility measurements close to zero and closure phases are sensitive to departures from circular symmetry (due to stellar spots, for instance). Both the $3 \mathrm{~T}$ and $4 \mathrm{~T}$ operations are therefore well adapted to stellar activity studies and/or spot detection on stellar surfaces. In various contexts, high angular resolution measurements provided by optical interferometry appears to be very relevant to the study of geometrical constraints on stellar spotty surfaces and to be very complementary to existing spectro-photometric techniques. As an example, this technique can be well suited to the detection of spots on exoplanet host stars and to accurately quantify the stellar noise that can mimic planetary signals in radial-velocity and/or transit methods when searching for exoplanets (Lagrange et al. 2010). Provided that high spectral resolution is coupled to long-baseline interferometry, another challenge is to be able to detect and image the abundance inhomogeneities of chemically peculiar stars, which will be an important step in more clearly understanding this peculiar class of objects and the physical processes that occur in a strong large-scale organized magnetic field.

\subsection{Other scientific goals}

Coupling high angular resolution to high spectral resolution is also of interest for kinematic studies that use emission lines. Being able to reconstruct velocity-resolved images is a powerful means of studying the complex environments of hot stars, permitting us to probe the geometry and kinematics of the dust and the gas that surrounds these objects or to provide constraints on the size, the inclination, or the position angle of the circumstellar disks. It could also provide information about the stellar rotation and the differential rotation for fast rotators (Zorec et al. 2011).

Binary star study will also greatly benefit from a combination of high spectral and high spatial resolutions. This is indeed the ideal way to achieve accurate measurements of mass and luminosity, which are the primary parameters of stellar evolution models.

Finally, it has been demonstrated that using spectral differential observables to reconstruct images greatly improves the quality of the reconstructed images, which are then astrometrically linked from wavelength-to-wavelength (Millour et al. 2011).

\section{Conclusion}

We have described the principles and the formalism of the spatio-spectral encoding of fringes. This mode is well adapted to multi-axial dispersed fringe instruments for optical longbaseline interferometry and allows us to simultaneously reduce the size of the optics to accommodate the beams, decrease the number of pixels that have to be read to correctly analyze all the fringe systems, and increase the SNR and thus the limiting magnitude of these instruments.

Thanks to the dual instrument capabilities of the CHARA array operating system, we have been able to use the visible spectrograph VEGA in parallel with the infrared instruments CLIMB and MIRC. A low-frequency group-delay tracking of fringes in the infrared has been shown to permit an excellent stabilization 

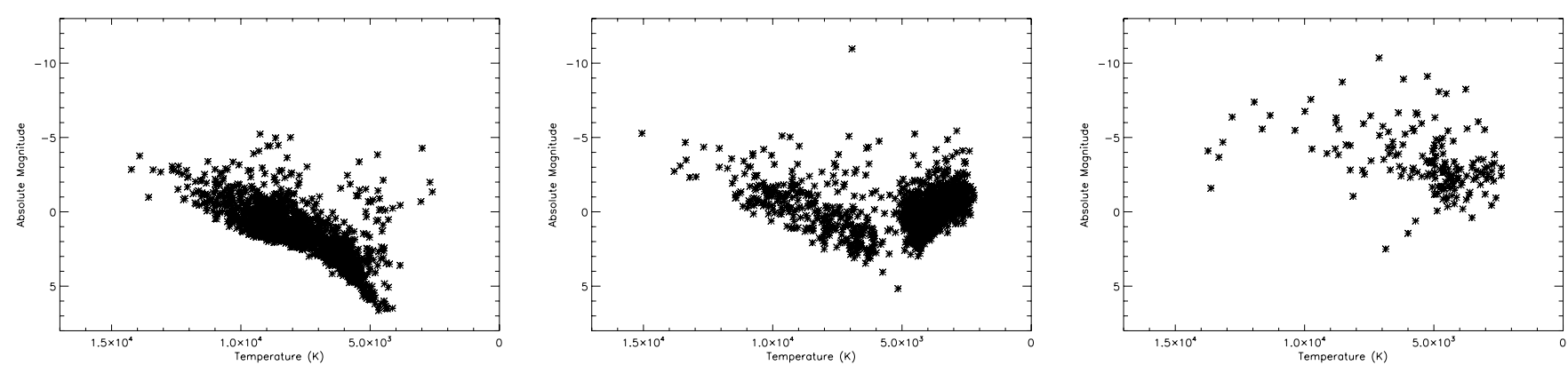

Fig. 11. HR diagrams of stars observable with VEGA in terms of accurate limb darkening measurements. Left: main sequence stars (luminosity class V); middle: giant stars (luminosity class III); right: supergiants stars (luminosity class I).

of the optical path difference at the level of $5 \mu \mathrm{m}$ over long periods of time, an achievement that is ideal for a high spectral dispersion instrument. Thus, observing faint visibilities in the visible becomes easier. It has permitted us to qualify the 3T- and 4T-mode of the VEGA spectrograph. The current performance of these modes has been evaluated in this paper, in terms of both reliability and precision in $V^{2}$, closure phase and differential visibility measurements.

Finally we have described the interesting astrophysical programs enabled by the combination of this original encoding mode and the particular configuration of the CHARA Array.

Acknowledgements. We wish to warmly thank Farrokh Vakili for fruitful discussions on the topic of this paper and for his continuous support to VEGA/CHARA.

VEGA is a collaboration between CHARA and OCA/LAOG/CRAL/LESIA that has been supported by the French programs PNPS and ASHRA, by INSU and by the Région PACA. The CHARA Array is operated with support from the National Science Foundation through grant AST-0908253, the W. M. Keck Foundation, the NASA Exoplanet Science Institute, and from Georgia State University. This research has made use of the Jean-Marie Mariotti Center SearchCal service ${ }^{3}$.

\section{References}

Berio, P., Stee, P., Vakili, F., et al. 1999, A\&A, 345, 203

Blazit, A., Rondeau, X., Thiébaut, E., et al. 2008, Appl. Opt., 47, 1141

Bonneau, D., Clausse, J., Delfosse, X., et al. 2006a, A\&A, 456, 789

Bonneau, D., Clausse, J.-M., Delfosse, X., et al. 2006b, A\&A, 456, 789

Chiavassa, A., Collet, R., Casagrande, L., \& Asplund, M. 2010, A\&A, 524, A93
Ciddor, P. E. 1996, Appl. Opt., 35, 1566

Colavita, M. M., Swain, M. R., Akeson, R. L., Koresko, C. D., \& Hill, R. J. 2004, PASP, 116, 876

Cornwell, T. J. 1987, A\&A, 180, 269

Coude du Foresto, V., Borde, P. J., Merand, A., et al. 2003, in SPIE Conf. Ser. 4838, ed. W. A. Traub, 280

Ireland, M. J., Mérand, A., ten Brummelaar, T. A., et al. 2008, SPIE Conf. Ser., 7013, 1

Kervella, P., Thévenin, F., Di Folco, E., \& Ségransan, D. 2004, A\&A, 426, 297

Koechlin, L., Lawson, P. R., Mourard, D., et al. 1996, Appl. Opt., 35, 3002

Labeyrie, A. 1975, ApJ, 196, L71

Lagrange, A., Desort, M., \& Meunier, N. 2010, A\&A, 512, A38

Le Bouquin, J., \& Tatulli, E. 2006, MNRAS, 372, 639

Matter, A., Vannier, M., Morel, S., et al. 2010, A\&A, 515, A69

Meilland, A., Stee, P., Vannier, M., et al. 2007, A\&A, 464, 59

Millour, F., Meilland, A., Chesneau, O., et al. 2011, A\&A, 526, A107

Monnier, J. D., Zhao, M., Pedretti, E., et al. 2008, SPIE Conf. Ser., 7013

Mourard, D., Bosc, I., Labeyrie, A., Koechlin, L., \& Saha, S. 1989, Nature, 342, 520

Mourard, D., Blazit, A., Bonneau, D., et al. 2006, SPIE Conf. Ser., 6268, 118

Mourard, D., Perraut, K., Bonneau, D., et al. 2008, SPIE Conf. Ser., 7013, 62

Mourard, D., Clausse, J. M., Marcotto, A., et al. 2009, A\&A, 508, 1073

Mourard, D., Tallon, M., Bério, P., et al. 2010, SPIE Conf. Ser., 7734, 11

Petrov, R. G., Malbet, F., Weigelt, G., et al. 2007, A\&A, 464, 1

Sturmann, J., Ten Brummelaar, T., Sturmann, L., \& McAlister, H. A. 2010, SPIE Conf. Ser., 7734, 104

Tallon-Bosc, I., Tallon, M., Thiébaut, E., et al. 2008, SPIE Conf. Ser., 7013, 44 ten Brummelaar, T. A., McAlister, H. A., Ridgway, S. T., et al. 2005, ApJ, 628, 453

Vakili, F., \& Koechlin, L. 1989, in SPIE Conf. Ser. 1130, ed. J.-P. Swings, 109 van Leeuwen, F. 2007, A\&A, 474, 653

Woan, G., \& Duffett-Smith, P. J. 1988, A\&A, 198, 375

Zorec, J., Frémat, Y., Domiciano de Souza, A., et al. 2011, A\&A, 526, A87

\footnotetext{
3 Available at http://www.jmmc. fr/searchcal co-developped by FIZEAU and LAOG, of the Jean-Marie Mariotti Center LITpro service co-developed by CRAL, LAOG and FIZEAU, and of CDS Astronomical Databases SIMBAD and VIZIER ${ }^{4}$
} 